\title{
Queixas de voz em agentes comunitários de saúde: correlação entre problemas gerais de saúde, hábitos de vida e aspectos vocais
}

\author{
Voice complaints in community health agents: correlation \\ between general health problems, lifestyle habits and vocal \\ aspects
}

Fabiana Gonçalves Cipriano ${ }^{1}$, Léslie Piccolotto Ferreira ${ }^{2}$

\begin{abstract}
RESUMO
Objetivo: Verificar as queixas de voz e correlacioná-las à autorreferência de problemas gerais de saúde, hábitos de vida e aspectos vocais, em um grupo de Agentes Comunitários de Saúde (ACS). Métodos: Participaram 28 ACS, os quais responderam a um questionário sobre dados pessoais, tempo de atuação como ACS, aspectos gerais de saúde, hábitos de vida e aspectos vocais. Os dados foram analisados estatisticamente. Resultados: Dos ACS participantes, 42,9\% relataram queixas de voz e as atribuíram às seguintes causas: exposição ao frio (30,7\%), infecção respiratória $(23,7 \%)$ e uso intensivo da voz (23,0\%). Foi observada associação significativa entre a presença de queixas de voz e as relacionadas a problemas emocionais $(\mathrm{p}=0,009)$ e problemas na coluna $(\mathrm{p}=0,038)$. Os sintomas vocais mais citados foram: rouquidão (33,3\%), falta de ar (32,1\%), falha na voz (14,2\%), voz grossa (14,2\%) e voz variando grossa/ fina $(14,2 \%)$. Em relação aos sintomas laringofaríngeos, os ACS mencionaram: garganta seca $(32,1 \%)$, cansaço ao falar $(32,1 \%)$, tosse seca $(28,5 \%)$, secreção na garganta (25\%) e ardor na garganta (21,4\%). Quando tais sintomas, vocais e laringofaríngeos, foram correlacionados com a presença de queixas de voz, foi verificada associação significativa com os sintomas vocais: rouquidão $(\mathrm{p}=0,001)$, falha na voz $(\mathrm{p}=0,024)$ e voz grossa $(\mathrm{p}=0,024)$. Conclusão: Houve expressiva porcentagem, dentre os ACS, de queixas de voz, caracterizadas, principalmente, pela presença de rouquidão, falha na voz e voz grossa. Problemas na coluna e emocionais mostraram-se associados à presença de queixas de voz.
\end{abstract}

Descritores: Distúrbios da voz; Fatores de risco; Pessoal de saúde; Saúde do trabalhador; Qualidade da voz; Estilo de vida; Pessoal de saúde

\section{INTRODUÇÃO}

O Programa Saúde da Família (PSF) foi proposto pelo Ministério da Saúde, em 1994, como estratégia de consolidação e reorganização da prática de assistência pública à saúde, com o objetivo de aprimorar e ampliar o atendimento à população(1).

Para tanto, o Programa conta com o trabalho de equipes multiprofissionais de saúde, cuja constituição básica inclui: um médico, um enfermeiro, dois auxiliares de enfermagem

Trabalho realizado no Curso de Especialização em Voz, Pontifícia Universidade Católica de São Paulo - PUC-SP - São Paulo (SP) - Brasil.

(1) Programa de Estudos Pós-Graduados em Fonoaudiologia da Pontifícia Universidade Católica de São Paulo - PUC-SP - São Paulo (SP), Brasil.

(2) Departamento de Fundamentos da Fonoaudiologia, Pontifícia Universidade Católica de São Paulo - PUC-SP - São Paulo (SP) - Brasil.

Endereço para correspondência: Fabiana Gonçalves Cipriano. R. Madre de Deus, 1285/11, Mooca, São Paulo (SP), Brasil, CEP: 03119-001. E-mail: fabianagcipriano@uol.com.br

Recebido em: 22/2/2010; Aceito em: 13/9/2010 e de quatro a seis Agentes Comunitários de Saúde (ACS). O suporte de atuação das equipes é constituído pelas Unidades Básicas de Saúde (UBS), que realizam visitas e internação domiciliar $^{(1)}$.

No entanto, algumas pesquisas ${ }^{(2,3)}$ observam que os ACS têm papel muito específico, que os diferencia dos demais membros da equipe de saúde: obrigatoriamente, devem residir em sua área de atuação, o que condiciona envolvimento pessoal diferenciado com a comunidade; por consequência, convivem com a realidade e as práticas de saúde do local onde habitam e trabalham, o que pressupõe maior identificação com a cultura, linguagem e costumes da sua própria comunidade.

Suas principais atribuições, de acordo com o Ministério da Saúde ${ }^{(1)}$, são: analisar as necessidades da comunidade; atuar em ações de prevenção de doenças e promoção da saúde, especialmente da criança, da mulher, do adolescente, do idoso e dos portadores de deficiência física e mental; participar das 
ações de saneamento básico e melhoria do ambiente e participar das reuniões da equipe de saúde e outros eventos de saúde junto à comunidade.

Entre as principais atividades de trabalho, estão incluídas entrevistas, visitas domiciliares, cadastramento das famílias, mapeamento da comunidade e reuniões comunitárias ${ }^{(4)}$.

Espera-se que o ACS tenha bom relacionamento com a comunidade, saiba trabalhar de acordo com princípios éticos, mantendo sigilo profissional e tenha facilidade de comunicação ${ }^{(5)}$. Nesta direção, destaca-se "a valorização do diálogo - a conversa, como instrumento de trabalho"(6).

$\mathrm{Na}$ Fonoaudiologia, merece destaque o trabalho desenvolvido com ACS na cidade de Jataí (GO), que se constituiu de dois encontros: no primeiro, o objetivo foi esclarecer o que é Fonoaudiologia e seu campo de atuação; e, no segundo, foram abordados dados referentes à anatomia e fisiologia vocal e cuidados com a $\mathrm{voz}^{(7)}$. A autora analisa a importância deste tipo de atuação, por parte do fonoaudiólogo, por considerar que a voz do ACS, na interlocução com os usuários, é importante para o desenvolvimento da sua função ${ }^{(7)}$.

Tendo em vista as peculiaridades existentes no trabalho dos ACS e a escassez de estudos sobre essa temática, o objetivo desta pesquisa é conhecer as queixas de voz e correlacionálas à autorreferência de problemas gerais de saúde, hábitos de vida e aspectos vocais, em um grupo de ACS atuantes no município de São Paulo.

\section{MÉTODOS}

\section{Local e população do estudo}

Foi selecionada, por conveniência, uma UBS localizada na região Leste do município de São Paulo, onde atuam 42 ACS. Foram critérios de inclusão: integrar a equipe da referida UBS e concordar em participar da pesquisa, por meio da assinatura do Termo de Consentimento Livre e Esclarecido (TCLE). A não finalização, por qualquer motivo, dos procedimentos da coleta de dados foi o único critério de exclusão. Houve perda de 14 participantes e a amostra final deste estudo foi constituída por $28(66,6 \%)$ ACS.

\section{Instrumento}

Como instrumento para a coleta de dados, elegeu-se o questionário Condições de Produção Vocal - Professor (CPV-P), proposto, prioritariamente, para o levantamento das condições de produção vocal de professores ${ }^{(8)}$. Trata-se de um questionário autorreferido, composto por 84 questões, divididas em seis partes.

$\mathrm{Na}$ adaptação para esta pesquisa permaneceram 30 questões, divididas em cinco partes, a saber: I- Identificação do questionário; II- Identificação do entrevistado (nome, nascimento, sexo e tempo de atuação como ACS); III- Aspectos gerais de saúde (presença de estresse no trabalho, problemas digestivos, na coluna, emocionais, respiratórios e de audição); IV- Hábitos de vida (tabagismo, consumo de bebida alcoólica, água e sono) e V- Aspectos vocais (queixas de voz, sintomas vocais e laringofaríngeos). Em sua maioria, as questões foram apresentadas em escala de frequência ( 0 . Nunca, 1 . Raramente, 2. às vezes, 3. sempre, 4. não sei).

\section{Procedimentos}

Esta pesquisa foi submetida e aprovada pelo Comitê de Ética em Pesquisa da Pontifícia Universidade Católica de São Paulo (parecer no 008/2007) e pelo Comitê de Ética em Pesquisa da Secretaria Municipal de Saúde de São Paulo (parecer $\mathrm{n}^{\circ}$ 279/2007).

Em contato com os ACS, em sua respectiva UBS de atuação, estes foram informados quanto ao objetivo, o caráter voluntário e a garantia de sigilo na participação da pesquisa. A seguir, os mesmos foram orientados quanto ao preenchimento do questionário e, avisados de que, em caso de dúvida, poderiam solicitar maiores esclarecimentos.

\section{Variáveis do estudo}

A variável dependente foi a presença de queixas de voz e as independentes foram: idade, gênero, tempo de atuação como ACS, presença de estresse no trabalho, problemas digestivos, na coluna, emocionais, respiratórios, de audição, tabagismo, consumo de bebida alcoólica, água e sono.

\section{Análise dos dados}

Para cada categoria analisada, foi considerada a situação de: "ausência", quando as frequências "nunca" e "raramente" foram assinaladas; "presença", quando assinaladas "às vezes" e "sempre"; e a frequência "não sei" foi desconsiderada da análise.

Os resultados foram duplamente digitados, em planilha específica, para excluir possíveis erros e, a seguir, foram submetidos à análise estatística. Nesta, foram utilizados testes paramétricos (teste-t) e não paramétricos (Mann-Whitney, Qui-quadrado e exato de Fisher), sendo considerados significativos os valores de p com nível $\leq 5 \%(0,05)$.

\section{RESULTADOS}

Dos 28 participantes, $25(89,3 \%)$ eram do gênero feminino e $3(10,7 \%)$ do masculino. A média de idade foi de 37,8 anos (mín=21; máx=70; DP=12,7 anos) e a média de tempo de atuação como ACS foi de aproximadamente quatro anos (mín=2 meses; máx=8 anos; DP=2,8 anos).

Com relação à presença de queixas de voz, 12(42,9\%) dos ACS referiram apresentá-la há cerca de três anos (mínimo de 3 e máximo de 4,5 anos; $\mathrm{DP}=1,8$ anos). Segundo os participantes, as principais causas das queixas são: exposição ao frio, infecção respiratória e uso intensivo da voz.

Não foi encontrada diferença entre grupos com e sem queixas de voz, levando-se em conta as variáveis: gênero, idade e tempo de atuação como ACS (Tabela 1).

Entre os ACS pesquisados, 23 fizeram referência à presença de estresse no trabalho. Quanto aos aspectos gerais de saúde, aproximadamente metade dos pesquisados relataram a presença de problemas emocionais e problemas na coluna. Para 
Tabela 1. Idade e tempo de atuação dos agentes comunitários de saúde

\begin{tabular}{lcccccc}
\hline Variável & $\mathrm{n}$ & Mínimo & Máximo & Média & DP & $\begin{array}{c}\text { Valor } \\
\text { de } \mathrm{p}\end{array}$ \\
\hline $\begin{array}{l}\text { Idade } \\
\text { (em anos) }\end{array}$ & 28 & 21 & 70 & 37,8 & 12,70 & 0,091 \\
$\begin{array}{l}\text { Tempo de } \\
\text { profissão } \\
\text { (em meses) }\end{array}$ & 28 & 2 & 96 & 49,1 & 34,66 & 0,403 \\
\hline
\end{tabular}

Teste de Mann-Whitney $(p \leq 0,05)$

Legenda: $\mathrm{DP}=$ desvio-padrão

os aspectos emocionais, a maioria não detalhou problema e quando o fez, a justificativa "tristeza" foi a mais mencionada. Entre os problemas na coluna, os mais citados foram "dor" e "desvio". Entre os problemas digestivos, os mais mencionados foram azia e gastrite e entre os problemas respiratórios, resfriados, rinite e sinusite (Tabela 2 ).

Em relação aos problemas de audição, as queixas mais citadas pelos ACS foram: incômodo a sons ou ruídos, zumbido e dificuldade para ouvir.

Quando os grupos com e sem queixas de voz foram comparados, levando-se em conta os problemas emocionais e de coluna, houve diferença determinada por problemas emocionais e problemas na coluna.

Entre os hábitos de vida, o fumo foi assinalado por $25 \%$ dos ACS; $14,2 \%$ mencionaram o uso social de bebida alcoólica, sendo que a maioria prefere os fermentados. Quando questionados sobre o consumo de água, foi verificado que $96,4 \%$ ingerem mais de um litro ao dia, e, entre estes, $66,6 \%$ optam

Tabela 2. Distribuição dos agentes comunitários de saúde, segundo a frequência de queixas de voz relacionada à autorreferência de problemas gerais de saúde, problemas de vias aéreas e de audição $(n=28)$

\begin{tabular}{|c|c|c|c|c|c|c|c|c|c|}
\hline \multirow{3}{*}{ Variável } & & & \multicolumn{6}{|c|}{ Queixas de voz } & \multirow{3}{*}{ Valor de $p$} \\
\hline & & & \multicolumn{3}{|c|}{ Ausência } & \multicolumn{3}{|c|}{ Presença } & \\
\hline & & & Não & Sim & Total & Não & Sim & Total & \\
\hline \multirow{12}{*}{$\begin{array}{l}\text { Problemas gerais } \\
\text { de saúde }\end{array}$} & \multirow{2}{*}{ Estresse } & $\mathrm{n}$ & 4 & 12 & 16 & 1 & 11 & 12 & \multirow{2}{*}{0,355} \\
\hline & & $\%$ & 25,0 & 75,0 & 57,2 & 8,4 & 91,6 & 42,8 & \\
\hline & \multirow{2}{*}{ Azia } & $\mathrm{n}$ & 12 & 4 & 16 & 6 & 6 & 12 & \multirow{2}{*}{0,243} \\
\hline & & $\%$ & 75,0 & 25,0 & 57,2 & 50,0 & 50,0 & 42,8 & \\
\hline & \multirow{2}{*}{ Refluxo } & $\mathrm{n}$ & 14 & 2 & 16 & 11 & 1 & 12 & \multirow{2}{*}{1,000} \\
\hline & & $\%$ & 87,5 & 12,5 & 57,5 & 91,7 & 8,3 & 42,5 & \\
\hline & \multirow{2}{*}{ Gastrite } & $\mathrm{n}$ & 14 & 2 & 16 & 10 & 2 & 12 & \multirow{2}{*}{1,000} \\
\hline & & $\%$ & 87,5 & 12,5 & 57,1 & 83,3 & 16,7 & 42,9 & \\
\hline & \multirow{2}{*}{ Coluna } & $\mathrm{n}$ & 11 & 4 & 15 & 4 & 8 & 12 & \multirow{2}{*}{$0,038^{*}$} \\
\hline & & $\%$ & 73,3 & 26,7 & 55,5 & 33,3 & 66,7 & 44,5 & \\
\hline & \multirow{2}{*}{ Emocional } & $\mathrm{n}$ & 12 & 4 & 16 & 3 & 9 & 12 & \multirow{2}{*}{$0,009^{*}$} \\
\hline & & $\%$ & 75,0 & 25,0 & 57,2 & 25,0 & 75,0 & 42,8 & \\
\hline \multirow{14}{*}{$\begin{array}{l}\text { Problemas das } \\
\text { vias aéreas }\end{array}$} & \multirow{2}{*}{ Rinite } & $n$ & 9 & 7 & 16 & 7 & 5 & 12 & \multirow{2}{*}{0,912} \\
\hline & & $\%$ & 56,2 & 43,8 & 57,2 & 58,3 & 41,7 & 42,8 & \\
\hline & \multirow{2}{*}{ Sinusite } & $\mathrm{n}$ & 12 & 4 & 16 & 8 & 4 & 12 & \multirow{2}{*}{0,691} \\
\hline & & $\%$ & 75,0 & 25,0 & 57,1 & 66,7 & 33,3 & 42,9 & \\
\hline & \multirow{2}{*}{ Amigdalite } & $\mathrm{n}$ & 14 & 2 & 16 & 8 & 4 & 12 & \multirow{2}{*}{0,354} \\
\hline & & $\%$ & 87,5 & 12,5 & 57,1 & 66,7 & 33,3 & 42,9 & \\
\hline & Faringite & $n$ & 13 & 3 & 16 & 9 & 3 & 12 & \\
\hline & rarıngite & $\%$ & 81,2 & 18,8 & 57,1 & 75,0 & 25,0 & 42,9 & 1,000 \\
\hline & I aringito & $\mathrm{n}$ & 15 & 1 & 16 & 11 & 1 & 12 & 1000 \\
\hline & Laringite & $\%$ & 93,8 & 6,2 & 57,1 & 91,7 & 8,3 & 42,9 & 1,000 \\
\hline & & $\mathrm{n}$ & 15 & 1 & 16 & 11 & 1 & 12 & \\
\hline & Bronquite & $\%$ & 93,8 & 6,2 & 57,1 & 91,6 & 8,3 & 42,9 & 1,000 \\
\hline & Recfriadn & $\mathrm{n}$ & 8 & 8 & 16 & 5 & 7 & 12 & 0662 \\
\hline & Restrlado & $\%$ & 50,0 & 50,0 & 57,1 & 41,7 & 58,3 & 42,9 & 0,662 \\
\hline & Dificuldade nara ouvir & $\mathrm{n}$ & 15 & 1 & 16 & 8 & 4 & 12 & 0133 \\
\hline & Diticuldade para ouvir & $\%$ & 93,8 & 6,2 & 57,1 & 66,7 & 33,3 & 42,9 & 0,133 \\
\hline & Dorde ouvide & $\mathrm{n}$ & 15 & 1 & 16 & 11 & 1 & 12 & 1000 \\
\hline & Dor de ouvido & $\%$ & 93,8 & 6,2 & 57,1 & 91,7 & 8,3 & 42,9 & 1,000 \\
\hline Problema de & Incômodo a sons ou & $\mathrm{n}$ & 10 & 6 & 16 & 5 & 7 & 12 & 0274 \\
\hline audição & ruídos & $\%$ & 62,5 & 37,5 & 57,1 & 41,7 & 58,3 & 42,9 & $0,2 / 4$ \\
\hline & 7umbido & $\mathrm{n}$ & 13 & 3 & 16 & 6 & 6 & 12 & 0.114 \\
\hline & & $\%$ & 81,2 & 18,8 & 57,1 & 50,0 & 50,0 & 42,9 & 0,114 \\
\hline & Tontura e/ou vertiaem & $\mathrm{n}$ & 13 & 3 & 16 & 7 & 5 & 12 & 0231 \\
\hline & romitura elou vertigem & $\%$ & 81,2 & 18,8 & 57,1 & 58,3 & 41,7 & 42,9 & 0,201 \\
\hline
\end{tabular}

* Valores significativos $(p \leq 0,05)-$ Teste de Fisher 
Tabela 3. Distribuição dos agentes comunitários de saúde, segundo a frequência de queixas de voz, relacionada a hábitos de vida ( $\mathrm{n}=28$ )

\begin{tabular}{|c|c|c|c|c|c|c|c|c|c|}
\hline \multirow{3}{*}{ Variável } & & & \multicolumn{6}{|c|}{ Queixas de voz } & \multirow{3}{*}{ Valor de $p$} \\
\hline & & & \multicolumn{3}{|c|}{ Ausência } & \multicolumn{3}{|c|}{ Presença } & \\
\hline & & & Não & Sim & Total & Não & Sim & Total & \\
\hline \multirow{14}{*}{ Hábitos de vida } & \multirow{2}{*}{ Cigarro } & $n$ & 11 & 5 & 16 & 10 & 2 & 12 & \multirow{2}{*}{0,662} \\
\hline & & $\%$ & 68,8 & 31,2 & 57,1 & 83,3 & 16,7 & 42,9 & \\
\hline & \multirow{2}{*}{ Álcool } & $\mathrm{n}$ & 14 & 2 & 16 & 10 & 2 & 12 & \multirow{2}{*}{1,000} \\
\hline & & $\%$ & 84,5 & 15,5 & 57,1 & 83,3 & 16,7 & 42,9 & \\
\hline & \multirow{2}{*}{ Água } & $\mathrm{n}$ & 1 & 15 & 16 & 0 & 12 & 12 & \multirow{2}{*}{1,000} \\
\hline & & $\%$ & 6,2 & 93,8 & 57,1 & 0,0 & 100,0 & 42,9 & \\
\hline & \multirow{2}{*}{ Outros líquidos } & $\mathrm{n}$ & 1 & 15 & 16 & 1 & 11 & 12 & \multirow{2}{*}{1,000} \\
\hline & & $\%$ & 6,2 & 93,8 & 57,1 & 8,3 & 91,7 & 42,9 & \\
\hline & \multirow{2}{*}{ Gelados } & $\mathrm{n}$ & 6 & 9 & 15 & 3 & 9 & 12 & \multirow{2}{*}{0,683} \\
\hline & & $\%$ & 40,0 & 60,0 & 55,5 & 25,0 & 75,0 & 44,5 & \\
\hline & \multirow{2}{*}{ Acorda à noite } & $\mathrm{n}$ & 4 & 12 & 16 & 2 & 10 & 12 & \multirow{2}{*}{0,673} \\
\hline & & $\%$ & 25,0 & 75,0 & 57,1 & 16,7 & 83,3 & 42,9 & \\
\hline & \multirow{2}{*}{ Acorda descansado } & $\mathrm{n}$ & 3 & 13 & 16 & 4 & 8 & 12 & \multirow{2}{*}{0,418} \\
\hline & & $\%$ & 38,8 & 61,2 & 57,1 & 33,3 & 66,7 & 42,9 & \\
\hline
\end{tabular}

Teste de Fisher $(p \leq 0,05)$

pela temperatura gelada. Os ACS informaram que dormem, em média, sete horas por noite, $78,5 \%$ costumam acordar durante a noite e $25 \%$ acordam cansados. Na comparação dos grupos com e sem queixas de voz levando-se em conta as variáveis relacionadas aos hábitos de vida, não foi encontrada associação significativa (Tabela 3 ).

Os sintomas vocais mais citados pelos ACS foram: rouquidão, falta de ar, falha na voz, voz grossa e voz variando grossa/fina. Em relação aos sintomas laringofaríngeos, os ACS mencionaram, principalmente, garganta seca. Quando foram comparados os indivíduos com e sem queixas de voz, levando-se em conta os sintomas vocais e laringofaríngeos, houve diferença determinada pelos sintomas vocais: rouquidão $(\mathrm{p}=0,001)$, falha na voz $(\mathrm{p}=0,024)$ e voz grossa $(\mathrm{p}=0,024)$ (Tabelas 4 e 5).

\section{DISCUSSÃO}

Nesta pesquisa, pôde-se notar um número superior de mulheres em relação ao de homens. Os estudos realizados junto aos ACS, à semelhança dos realizados com professores ${ }^{(9,10)}$, demonstram o predomínio de mulheres atuantes nesta ocupação. Quanto às questões de produção vocal, a literatura indica ${ }^{(11)}$ que a mulher apresenta maior predisposição ao desenvolvimento de alterações de voz, devido, principalmente, às dimensões reduzidas da laringe. No entanto, na comparação dos grupos com e sem queixa, levando-se em conta a variável gênero, não foi evidenciada diferença entre os grupos, fato que aponta para direção oposta de pesquisas realizadas com professores ${ }^{(12,13)}$.

A média de idade encontrada, nesta população, é próxima à da pesquisa realizada junto aos ACS atuantes em Porto Ale-

Tabela 4. Distribuição dos agentes comunitários de saúde, segundo a frequência de queixas de voz, relacionada aos sintomas vocais ( $\mathrm{n}=28$ )

\begin{tabular}{|c|c|c|c|c|c|c|c|c|c|}
\hline \multirow{3}{*}{ Variável } & & & \multicolumn{6}{|c|}{ Queixas de voz } & \multirow{3}{*}{ Valor de $p$} \\
\hline & & & \multicolumn{3}{|c|}{ Ausência } & \multicolumn{3}{|c|}{ Presença } & \\
\hline & & & Não & $\operatorname{Sim}$ & Total & Não & Sim & Total & \\
\hline \multirow{16}{*}{ Sintomas vocais } & \multirow{2}{*}{ Rouquidão } & $\mathrm{n}$ & 15 & 1 & 16 & 3 & 8 & 11 & \multirow{2}{*}{$0,001^{*}$} \\
\hline & & $\%$ & 93,8 & 6,2 & 59,2 & 27,2 & 72,8 & 40,8 & \\
\hline & \multirow{2}{*}{ Perda da voz } & $\mathrm{n}$ & 16 & 0 & 16 & 10 & 2 & 12 & \multirow{2}{*}{0,175} \\
\hline & & $\%$ & 61,5 & 0,0 & 57,1 & 83,3 & 16,7 & 42,9 & \\
\hline & \multirow{2}{*}{ Falha na voz } & $\mathrm{n}$ & 16 & 0 & 16 & 8 & 4 & 12 & \multirow{2}{*}{$0,024^{*}$} \\
\hline & & $\%$ & 100,0 & 0,0 & 57,1 & 66,7 & 33,3 & 42,9 & \\
\hline & \multirow{2}{*}{ Falta de ar } & $\mathrm{n}$ & 12 & 4 & 16 & 7 & 5 & 12 & \multirow{2}{*}{0,432} \\
\hline & & $\%$ & 75,0 & 25,0 & 57,1 & 58,3 & 41,7 & 42,9 & \\
\hline & \multirow{2}{*}{ Voz fina } & $\mathrm{n}$ & 16 & 0 & 16 & 11 & 1 & 12 & \multirow{2}{*}{0,429} \\
\hline & & $\%$ & 100,0 & 0,0 & 57,1 & 91,7 & 8,3 & 42,9 & \\
\hline & \multirow{2}{*}{ Voz grossa } & $\mathrm{n}$ & 16 & 0 & 16 & 8 & 4 & 12 & \multirow{2}{*}{$0,024^{*}$} \\
\hline & & $\%$ & 100,0 & 0,0 & 57,1 & 66,7 & 33,3 & 42,9 & \\
\hline & \multirow{2}{*}{ Voz variando grossa/fina } & $\mathrm{n}$ & 14 & 2 & 16 & 10 & 2 & 12 & \multirow{2}{*}{1,000} \\
\hline & & $\%$ & 87,5 & 12,5 & 57,1 & 83,3 & 16,7 & 42,9 & \\
\hline & \multirow{2}{*}{ Voz fraca } & $\mathrm{n}$ & 16 & 0 & 16 & 10 & 2 & 12 & \multirow{2}{*}{0,175} \\
\hline & & $\%$ & 100,0 & 0,0 & 57,1 & 83,3 & 16,7 & 42,9 & \\
\hline
\end{tabular}

*Valores significativos $(p \leq 0,05)-$ Teste de Fisher 
Tabela 5. Distribuição dos agentes comunitários de saúde, segundo a frequência de queixas de voz, relacionada aos sintomas laringofaríngeos $(\mathrm{n}=28)$

\begin{tabular}{|c|c|c|c|c|c|c|c|c|c|}
\hline \multirow{3}{*}{ Variável } & & & \multicolumn{6}{|c|}{ Queixas de voz } & \multirow{3}{*}{ Valor de $p$} \\
\hline & & & \multicolumn{3}{|c|}{ Ausente } & \multicolumn{3}{|c|}{ Presente } & \\
\hline & & & Não & Sim & Total & Não & $\operatorname{Sim}$ & Total & \\
\hline \multirow{28}{*}{$\begin{array}{l}\text { Sintomas } \\
\text { laringofaríngeos }\end{array}$} & \multirow{2}{*}{ Picada na garganta } & $\mathrm{n}$ & 16 & 0 & 16 & 9 & 3 & 12 & \multirow{2}{*}{0,067} \\
\hline & & $\%$ & 100,0 & 0,0 & 57,1 & 75,0 & 25,0 & 42,9 & \\
\hline & \multirow{2}{*}{ Areia na garganta } & $\mathrm{n}$ & 14 & 2 & 16 & 10 & 2 & 12 & \multirow{2}{*}{1,000} \\
\hline & & $\%$ & 87,5 & 12,5 & 57,1 & 83,3 & 16,7 & 42,9 & \\
\hline & \multirow{2}{*}{ Bola na garganta } & $\mathrm{n}$ & 12 & 4 & 16 & 12 & 0 & 12 & \multirow{2}{*}{0,113} \\
\hline & & $\%$ & 75,0 & 25,0 & 57,5 & 100,0 & 0,0 & 42,5 & \\
\hline & \multirow{2}{*}{ Pigarro } & $\mathrm{n}$ & 12 & 4 & 16 & 10 & 2 & 12 & \multirow{2}{*}{0,673} \\
\hline & & $\%$ & 75,0 & 25,0 & 57,1 & 83,3 & 16,7 & 42,9 & \\
\hline & \multirow{2}{*}{ Tosse seca } & $\mathrm{n}$ & 12 & 4 & 16 & 8 & 4 & 12 & \multirow{2}{*}{0,691} \\
\hline & & $\%$ & 75,0 & 25,0 & 57,1 & 75,0 & 25,0 & 42,9 & \\
\hline & \multirow{2}{*}{ Tosse com catarro } & $\mathrm{n}$ & 14 & 2 & 16 & 10 & 2 & 12 & \multirow{2}{*}{1,000} \\
\hline & & $\%$ & 84,5 & 15,5 & 57,1 & 83,3 & 16,7 & 42,9 & \\
\hline & \multirow{2}{*}{ Dor ao falar } & $\mathrm{n}$ & 15 & 1 & 16 & 10 & 2 & 12 & \multirow{2}{*}{0,560} \\
\hline & & $\%$ & 93,8 & 6,2 & 57,1 & 83,3 & 16,7 & 42,9 & \\
\hline & \multirow{2}{*}{ Dor ao engolir } & $\mathrm{n}$ & 16 & 0 & 16 & 10 & 2 & 12 & \multirow{2}{*}{0,175} \\
\hline & & $\%$ & 100,0 & 0,0 & 57,1 & 83,3 & 16,7 & 42,9 & \\
\hline & \multirow{2}{*}{ Dificuldade para engolir } & $\mathrm{n}$ & 15 & 1 & 16 & 9 & 3 & 12 & \multirow{2}{*}{0,285} \\
\hline & & $\%$ & 93,8 & 6,2 & 57,1 & 75,0 & 25,0 & 42,9 & \\
\hline & \multirow{2}{*}{ Ardor na garganta } & $\mathrm{n}$ & 13 & 3 & 16 & 9 & 3 & 12 & \multirow{2}{*}{1,000} \\
\hline & & $\%$ & 81,2 & 18,8 & 57,1 & 75,0 & 25,0 & 42,9 & \\
\hline & \multirow{2}{*}{ Secreção na garganta } & $\mathrm{n}$ & 12 & 4 & 16 & 9 & 3 & 12 & \multirow{2}{*}{1,000} \\
\hline & & $\%$ & 75,0 & 25,0 & 57,1 & 75,0 & 25,0 & 42,9 & \\
\hline & \multirow{2}{*}{ Garganta seca } & $\mathrm{n}$ & 12 & 4 & 16 & 7 & 5 & 12 & 0132 \\
\hline & & $\%$ & 75,0 & 25,0 & 57,1 & 58,3 & 41,7 & 42,9 & 0,402 \\
\hline & Cancaco an falar & $\mathrm{n}$ & 13 & 3 & 16 & 6 & 6 & 12 & 0114 \\
\hline & Cansaço ao talar & $\%$ & 81,2 & 18,8 & 57,1 & 50,0 & 50,0 & 42,9 & 0,174 \\
\hline & Esforco an falar & $\mathrm{n}$ & 16 & 0 & 16 & 9 & 3 & 12 & 0067 \\
\hline & & $\%$ & 100,0 & 0,0 & 57,1 & 75,0 & 25,0 & 42,9 & 0,067 \\
\hline
\end{tabular}

Teste de Fisher $(p \leq 0,05)$

gre $(\mathrm{RS})^{(9)}$, entre trinta e quarenta e nove anos. Em geral, há uma diminuição da eficiência respiratória nas pessoas com o aumento da idade ${ }^{(14)}$. Esta, por sua vez, pode influenciar de maneira negativa o controle da respiração e, portanto, o controle vocal $^{(11)}$. Alterações da laringe, como a atrofia dos músculos laríngeos, redução da elasticidade dos ligamentos e flacidez das pregas vocais, têm sido descritas na literatura ${ }^{(11)}$ e estes fatores têm sustentado a explicação do aumento da soprosidade, fadiga vocal, aumento de tremores na voz e variação no controle da frequência ${ }^{(11)}$. No caso das mulheres, a menopausa ocasiona a perda dos hormônios femininos; este fato, somado aos fatores relacionados à idade, pode ter um efeito direto sobre a laringe, como atrofia do músculo laríngeo, enrijecimento das cartilagens laríngeas, espessamento da prega vocal e perda das fibras elásticas e de colágeno ${ }^{(11)}$.

A média de tempo de ocupação encontrada nesta população é próxima à dos atuantes de Santa Maria $(\mathrm{RS})^{(10)}$, que variou entre um e sete anos. A inserção do ACS no âmbito do SUS ocorreu a partir de 1991, com a criação do Programa de Agentes Comunitários de Saúde (PACS). Por sua vez, a implantação do PSF, em 1994, intensificou a entrada destes trabalhadores no Sistema, fato que justifica a média de tempo de ocupação encontrada em ambas as pesquisas.

A diferença entre os grupos com e sem queixa, determinada pela referência a problemas na coluna e à presença de queixas de voz, pode ser esclarecida na pesquisa sobre cargas de trabalho* a que os ACS estão submetidos ${ }^{(10)}$. Foram identificadas cargas físicas, químicas, orgânicas, mecânicas e, principalmente, cargas psíquicas ${ }^{(10)}$. Quanto às cargas mecânicas, as queixas mais frequentes foram as longas caminhadas diárias que realizam, em decorrência das visitas domiciliares, e o peso das mochilas, às vezes, acrescido ao peso da balança, para pesagem das crianças ${ }^{(10)}$. Também destacaram dores na coluna, nas pernas e dores de cabeça ${ }^{(10)}$. Sintomas semelhantes foram encontrados entre os ACS desta pesquisa, como: problemas na coluna, dores e desvio na coluna e dores de cabeça.

De acordo com pesquisa realizada junto a 100 teleoperadores no município de São Paulo ${ }^{(15)}$, e que fez uso do mesmo instrumento utilizado neste estudo, a maior parte deles apresentou queixas referentes a problemas na coluna. As autoras

\footnotetext{
* Entende-se por cargas de trabalho o conjunto de esforços desenvolvidos, pelos trabalhadores, para atender às exigências do trabalho, o qual envolve demandas
} físicas, cognitivas e psicoafetivas (emocionais) ${ }^{(10)}$. 
concluíram que tais queixas podem relacionar-se com o tempo de trabalho realizado numa mesma posição e com o mobiliário inadequado. Estes achados corroboram pesquisa ${ }^{(10)}$ que aponta as posições incômodas e incorretas que os ACS assumem durante o trabalho, além da necessidade de permanecerem em posições incorretas durante as visitas domiciliares, uma vez que, em alguns casos, há falta de bancos ou cadeiras.

Ao mudar ou fixar-se em determinada posição, a musculatura do pescoço e dos ombros tende ao enrijecimento, podendo causar tensão, dor e interferir na produção vocal ${ }^{(15)}$. $\mathrm{O}$ excesso de tensão muscular, principalmente nas regiões da face, pescoço e ombros, pode impactar a mobilidade natural da laringe durante a fonação e a projeção adequada da voz.

Outra associação significativa observada diz respeito à autorreferência de problemas emocionais e à presença de queixas de voz. Diante deste achado, cabe ressaltar a especificidade da atividade de trabalho dos $\mathrm{ACS}^{(16)}$, uma vez que, obrigatoriamente, devem residir na área em que atuam. Por conseguinte, são abordados pela comunidade beneficiada fora do expediente de trabalho, por diferentes questões (dúvidas, orientações, agendamento de consultas, entre outros), seja na própria residência, nas ruas e/ou nos espaços coletivos. Tal situação predispõe estes trabalhadores a intenso envolvimento pessoal e desgaste emocional ${ }^{(17)}$.

A pesquisa anteriormente $\operatorname{citada}^{(17)}$ revela, ainda, a existência de aspectos precários e carentes de solução nos serviços públicos de saúde, os quais causam sofrimento adicional aos ACS. O contato próximo e ininterrupto com a comunidade acaba, muitas vezes, por estreitar o vínculo e a responsabilidade dos ACS com as famílias beneficiadas, o que os faz sentiremse, de certa forma, responsáveis pelo sofrimento dos usuários.

Com relação aos efeitos da emoção sobre a voz, autoras ${ }^{(18)}$ mencionam que alterações do estado emocional podem interferir na produção vocal e, inclusive, na produção da fala, nos parâmetros de coordenação pneumofonoarticulatória, vícios de linguagem e inteligibilidade de fala. Outros autores ${ }^{(11,19)}$ explicitam que dificuldades emocionais predispõem a determinadas alterações fisiológicas, entre elas o aumento no fluxo sanguíneo, na frequência respiratória e no tônus muscular laríngeo, fatores estes que podem interferir na produção vocal.

Quanto à presença de sintomas vocais nos grupos com e sem queixas de voz constatou-se o efeito diferencial de rouquidão, falha na voz e voz grossa.

A rouquidão indica, entre outros, agressão à laringe e às pregas vocais, devido, principalmente, ao uso excessivo e/ou inadequado da $\mathrm{voz}^{(20)}$. Sugere, ainda, a presença de refluxo gastroesofágico (RGE), definido como o retorno passivo do conteúdo gástrico para o esôfago, independente de sua etiologia, e pode ocorrer em circunstâncias fisiológicas ou patológicas ${ }^{(21)}$. Em circunstâncias patológicas é denominado RGE patológico ou doença do refluxo gastroesofágico (DRGE), sendo uma condição menos comum e de prognóstico mais grave, além da necessidade de abordagens diagnóstica e terapêutica diferentes ${ }^{(21)}$. Os principais sinais e sintomas da doença consistem em queimação no esôfago; azia; regurgitação de alimentos; excesso de salivação; sensação de corpo estranho ou bola na garganta; má respiração; dor de garganta; tosse seca; rouquidão (principalmente pela manhã); pigarro constante; dificuldade para engolir e, nos casos mais severos, pneumonia aspirativa ${ }^{(14,22)}$, sendo que alguns destes sintomas foram citados pela população estudada.

Quando o conteúdo gástrico alcança a laringe, é denominado refluxo laringofaríngeo ${ }^{(22)}$. Nesta situação, por ser o conteúdo gástrico extremamente irritativo, é possível encontrar a parte posterior da laringe avermelhada, com irritação e lesões nas pregas vocais, sendo o granuloma a manifestação mais frequente ${ }^{(14,22)}$.

A este respeito, pesquisa ${ }^{(23)}$ destaca que condições inadequadas da organização do trabalho colaboram para o consumo de alimentos inapropriados e ausência de horário regular das refeições, predispondo o trabalhador a problemas digestivos, que, por sua vez, podem repercutir em sua saúde e bem-estar vocal.

Assim como a rouquidão, os sintomas vocais falha na voz e voz grossa, de acordo com a literatura ${ }^{(15,20,24)}$ sugerem, entre outros, o uso excessivo e/ou inadequado da voz, além do hábito de gritar e falar em intensidade elevada. Ainda, condições inadequadas do ambiente de trabalho (como, por exemplo: presença de poeira; fumaça; produtos químicos; variações climáticas; entre outras) podem contribuir para a ocorrência desses sintomas vocais, entre os ACS.

Diante disso, outras autoras ${ }^{(10)}$ reconhecem que, em decorrência das visitas domiciliares, os ACS estão expostos a variações de temperatura (muito altas, no verão, e muito baixas, no inverno), além da poeira e fumaça provenientes das ruas e dos automóveis, especialmente no município de São Paulo.

Finalmente, cabe considerar a expressiva porcentagem de queixas vocais entre os ACS (42,9\%). Na sua maioria, num primeiro momento, tais queixas podem ser vistas como relacionadas, prioritariamente, aos problemas gerais de saúde e hábitos de vida dos pesquisados. Numa leitura mais atenta, no entanto, percebe-se que essas queixas estão intimamente relacionadas às adversidades do ambiente (como, por exemplo: poeira, fumaça, variações climáticas, entre outras) e, principalmente, à organização do trabalho (como, por exemplo: posições incômodas e incorretas durante a atividade, residir na área de atuação, intenso envolvimento pessoal com a comunidade beneficiada, entre outras) e, portanto, contribuem para a causalidade sobredeterminada das alterações de voz.

As pesquisas no campo da Fonoaudiologia, em particular as relacionadas aos profissionais da voz (ou seja, aqueles que têm na voz um importante instrumento de trabalho), em sua maioria, visam caracterizar o perfil vocal de determinado grupo de trabalhadores. Com base nos resultados obtidos, propõem intervenções que, frequentemente, priorizam as questões relacionadas aos aspectos individuais e biológicos, somadas aos hábitos vocais.

Propõe-se aqui um olhar mais amplo, por parte do fonoaudiólogo, direcionado à multicausalidade das alterações de voz, e não centrado apenas na produção vocal, mas também no modo de organização do processo de trabalho ${ }^{(25)}$ a que estes sujeitos estão submetidos. Essa pesquisa trouxe à luz alguns indicadores que podem auxiliar o redimensionamento da questão.

Para tanto, sugere-se futuros estudos com a ampliação do número de ACS, no sentido de compreender aspectos que são 
recorrentes nesta população. Esses achados poderão contribuir para que o fonoaudiólogo possa aprimorar sua prática, junto aos ACS, não somente por meio da atuação terapêutica individual, mas, também de ações que visem à promoção do bem-estar vocal e a prevenção de alterações de voz.

\section{CONCLUSÃO}

Nesta pesquisa, realizada junto a um grupo de ACS atuan- tes na região Leste do município de São Paulo, foi verificada presença de queixas de voz em porcentagem maior do que o observado na população em geral. Segundo os ACS, as principais causas que justificam tais queixas são: exposição ao frio, uso intensivo da voz e infecção respiratória.

Problemas na coluna e emocionais mostraram-se responsáveis pela referência à presença de queixas de voz, caracterizadas, principalmente, pela presença de rouquidão, falha na voz e voz grossa.

\begin{abstract}
Purpose: To verify vocal complaints and correlate them to self reported general health problems, lifestyle habits and vocal habits in a group of Community Health Agents (CHA). Methods: The subjects of this study were 28 CHA, who answered a questionnaire concerning their personal data, work situation, general health aspects, and vocal aspects and habits. Data was statistically analyzed. Results: From all the participants, $42.9 \%$ reported vocal complaints, and attributed them to the following causes: exposure to cold temperatures (30.7\%), respiratory infections (23.7\%) and intensive vocal use (20.3\%). It was observed significant correlation between the presence of self reported vocal complaints and those related to emotional issues $(\mathrm{p}=0.009)$ and back problems $(\mathrm{p}=0.038)$. The vocal symptoms most frequently reported were: hoarseness (33.3\%), shortage of breath (32.1\%), voice breaks (14.2\%), low-pitched voice (14.2\%), and voice varying between high and low pitches (14.2\%). Regarding laryngopharyngeal symptoms, CHA reported: dry throat $(32.1 \%)$, tiredness when speaking $(32.1 \%)$, dry cough $(28.5 \%)$, throat secretions $(25 \%)$, and burning throat (21.4\%). When these vocal and laryngopharyngeal symptoms were correlated to the presence of self-reported vocal complaints, it was verified a significant association with the following vocal symptoms: hoarseness $(\mathrm{p}=0.001)$, voice breaks $(\mathrm{p}=0.024)$, and low-pitched voice $(\mathrm{p}=0.024)$. Conclusion: There was a high percentage of CHAs who reported voice complaints, which were characterized mainly by hoarseness, voice breaks and low-pitched voice. Back problems and emotional issues were associated to the presence of voice complaints.
\end{abstract}

Keywords: Voice disorders; Risk factors; Health personnel; Occupational health; Voice quality; Life style; Health personnel

\title{
REFERÊNCIAS
}

1. Brasil. Ministério da Saúde. Secretaria de Assistência à Saúde. Coordenação de Saúde da Comunidade. Saúde da Família: uma estratégia para a reorientação do modelo assistencial. Brasília: Ministério da Saúde; 1997.

2. Sousa MF. Agentes Comunitários de Saúde (ACS): uma estratégia revolucionária em risco. Saúde Coletiva. 2008;4(19): 6.

3. Duarte LR, Silva DSJR, Cardoso SH. Construindo um programa de educação com agentes comunitários de saúde. Interface Comun Saúde Educ. 2007;11(23):439-47.

4. Kluthcovsky ACGC, Takayanagui AMM. Community health agent: a literature review. Rev Latinoam Enferm. 2006;14(6):957-63.

5. Martines WRV, Chaves EC. Vulnerabilidade e sofrimento no trabalho do agente comunitário de saúde no Programa Saúde da Família. Rev Esc Enferm USP. 2007;41(3):426-33.

6. Silva JA, Dalmaso ASW. O agente comunitário de saúde e suas atribuições: os desafios para os processos de formação de recursos humanos em saúde. Interface Comun Saúde Educ. 2002;6(10):75-83.

7. Vasconcelos IA. Fonoaudiologia e Agentes de Saúde: ação preventiva. In: Ferreira LP, Andrada e Silva MA, organizadoras. Saúde vocal: práticas fonoaudiológicas. São Paulo: Roca; c2002. p. 33-8.

8. Ferreira LP, Giannini SPP, Latorre MRDO, Zenari MS. Distúrbio de voz relacionado ao trabalho: proposta de instrumento para avaliação de professores. Distúrb Comun. 2007;19(1):127-36.

9. Ferraz L, Aerts DRGC. O cotidiano de trabalho do agente comunitário de saúde no PSF em Porto Alegre. Ciênc Saúde Coletiva. 2005;10(2):347-55.

10. Trindade LL, Gonzales RMB, Beck CLC, Lautert L. Cargas de trabalho entre os agentes comunitários de saúde. Rev Gaúch Enferm. 2007;28(4):473-9.

11. Freeman M, Fawcus M. Distúrbios da voz e seu tratamento. 4a ed. São Paulo: Santos; 2005.

12. Ferreira LP, Benedetti PH. Condições de produção vocal de professores de deficientes auditivos. Rev CEFAC. 2007;9(1):79-89.

13. Ferreira LP, Giannini SPP, Figueira S, Silva EE, Karmann DF, Souza TMT. Condições de produção vocal de professores da Prefeitura do Município de São Paulo. Distúrb Comun. 2003;14(2):275-307.

14. Boone DR. Inimigos biológicos da voz profissional. Pró-Fono. 1992;4(2):3-8.

15. Ferreira LP, Akutsu CM, Luciano P, Viviano NDAG. Condições de produção vocal de teleoperadores: correlação entre questões de saúde, hábitos e sintomas vocais. Rev Soc Bras Fonoaudiol. 2008;13(4):307-15.

16. Bornstein VJ, Stotz EN. Concepções que integram a formação e o processo de trabalho dos agentes comunitários de saúde: uma revisão da literatura. Ciênc Saúde Coletiva. 2008;13(1):259-68.

17. Jardim TA, Lancman S. Aspectos subjetivos do morar e trabalhar na mesma comunidade: a realidade vivenciada pelo agente comunitário de saúde. Interface Comun Saúde Educ. 2009;13(28):123-35.

18. Barbosa RA, Friedman S. Emoção: efeitos sobre a voz e a fala na situação em público. Distúrb Comun. 2007;19(3):325-36.

19. Steifert E, Kollbrunner J. Stress and distress in non-organic voice disorders. Swiss Med Wkly. 2005;135:387-97.

20. Pena JJ, Servilha EAM. Tipificação de sintomas relacionados à voz e sua produção em professores identificados com ausência de alteração vocal na avaliação fonoaudiológica. In: XIV Encontro de Iniciação Científica 
da PUC-Campinas - 29 e 30 de setembro de 2009. Anais. Campinas: PUC-Campinas; 2009.

21. Magalhães PVS, Appolinário JCB, Bacaltchuk J, Mota Neto JIS. Revisão sistemática e metanálise do uso de procinéticos no refluxo gastroesofágico e na doença do refluxo gastroesofágico em Pediatria. Rev Paul Pediatr. 2009;27(3):236-42.

22. Behlau M, Pontes P. Higiene vocal: cuidando da voz. Rio de Janeiro: Revinter; c2001. 3a ed. ampl. e atual. Higiene vocal e fatores de risco para a voz. p. 21-42.

23. Ferreira LP, de Oliveira Latorre Mdo R, Pinto Giannini SP, de Assis Moura Ghirardi AC, de França e Karmann D, Silva EE, Figueira S. Influence of abusive vocal habits, hydration, mastication, and sleep in the occurrence of vocal symptoms in teacher. J Voice. 2010;24(1):86-92.

24. Penteado RZ, Gonçalves CGO, Marques JM, Costa DD. Trabalho e saúde em motoristas de caminhão no interior de São Paulo. Saúde Soc. 2008;17(4):35-45.

25. Minayo Gomes C, Thedim-Costa SMF. A construção do campo da saúde do trabalhador: percurso e dilemas. Cad Saúde Pública $=$ Rep Public Health. 1997;13(Supl 2):21-32.

26. Roy N, Merril RM, Thibeault S, Gray SD, Smith EM. Voice disorders in teachers and the general population: effects on work performance, attendance, and future career choices. J Speech Lang Hear Res. 2004;47(3):542-51. 